\title{
APPROXIMATING A HARMONIZABLE ISOTROPIC RANDOM FIELD ${ }^{1}$
}

\author{
RANDALL J. SWIFT \\ Western Kentucky University \\ Department of Mathematics \\ Bowling Green, KY 42101 USA \\ E-mail: randall.swift@wku.edu
}

(Received June, 1999; Revised June, 2000)

\begin{abstract}
The class of harmonizable fields is a natural extension of the class of stationary fields. This paper considers a stochastic series approximation of a harmonizable isotropic random field. This approximation is useful for numerical simulation of such a field.
\end{abstract}

Key words: Harmonizable Random Field, Isotropy.

AMS subject classifications: $60 \mathrm{G} 12,60 \mathrm{G} 35$.

\section{Introduction}

In recent years the study of harmonizable processes has played a central role in the development of the theory of nonstationary processes. Crucial to this development is the pioneering work of Chang and Rao [1] on bimeasures and Morse-Transue integration. Their paper set the stage for the recent advances in the theory. A recent account of the development of harmonizable processes and some of their applications may be found in Swift [5]. That paper also contains a detailed bibliography of the existing work on harmonizable processes.

In this paper, a stochastic series approximation of a harmonizable isotropic random field is considered.

\section{Background}

Let $(\Omega, \Sigma, P)$ be a probability space. For $p \geq 1$, define $L_{0}^{p}(P)$ to be the set of all centered complex valued $f \in L^{p}(\Omega, \Sigma, P)$, that is $E(f)=0$, where $E(f)=\int_{\Omega} f(\omega) d P(\omega)$ is the expectation. We will consider second order random fields, more specifically, mappings $X: \mathbb{R}^{n} \rightarrow L_{0}^{2}(P)$.

\footnotetext{
${ }^{1}$ Presented at the Fifth International Conference on Combinatorics, Statistics, Pattern Recognition and Related Areas, Mysore India, December 28-30, 1998.
} 
A random field $X(t)$ is (weakly) stationary if its covariance function

$$
r(\boldsymbol{s}, \boldsymbol{t})=E(X(s) \overline{X(\boldsymbol{t}))}
$$

is translation invariant and continuous. Thus $r(\boldsymbol{s}, \boldsymbol{t})=\widetilde{r}(\boldsymbol{s}-\boldsymbol{t})$ and is also positive definite. The power of the stationary assumption is seen in the following classical theorem of Bochner.

Theorem 2.1: If $\tilde{r}(\cdot)$ is a continuous stationary covariance then there exists a unique bounded Borel measure $F: \mathbb{R}^{n} \rightarrow \mathbb{C}$ such that

$$
\tilde{r}(\boldsymbol{t})=\int_{\mathbb{R}^{n}} e^{i \boldsymbol{\lambda} \cdot \boldsymbol{t}} d \boldsymbol{F}(\boldsymbol{\lambda}) .
$$

This representation brings to bear the powerful Fourier analytic methods in the study of stationary processes and fields.

A motivation for the concept of harmonizability is to enlarge the applications of stationary processes and fields while retaining the Fourier analytic methods. This notion is seen in the following definition.

Definition 2.1: A random field $X: \mathbb{R}^{n} \rightarrow L_{0}^{2}(P)$ is weakly harmonizable if its covariance $r(\cdot, \cdot)$ is expressible as

$$
r(\boldsymbol{s}, \boldsymbol{t})=\int_{\mathbb{R}^{n}} \int_{\mathbb{R}^{n}} e^{i \boldsymbol{\lambda} \cdot \boldsymbol{s}-i \boldsymbol{\lambda}^{\prime} \cdot \boldsymbol{t}} d F\left(\boldsymbol{\lambda}, \boldsymbol{\lambda}^{\prime}\right)
$$

where $F: \mathbb{R}^{n} \times \mathbb{R}^{n} \rightarrow \mathbb{C}$ is a positive semi-definite bimeasure of bounded Fréchet variation.

A random field, $X(\cdot)$, is strongly harmonizable if the bimeasure $F(\cdot, \cdot)$ in (1) extends to a complex measure and hence is of bounded Vitali variation. Random fields often admit an additional property:

Definition 2.2: A random field $X: \mathbb{R}^{n} \rightarrow L^{2}(P)$ with mean $m(\cdot)$ and covariance $r(\cdot, \cdot)$ is isotropic if for each orthogonal matrix $g$ acting on $\mathbb{R}^{n}$, one has

$$
m(g \boldsymbol{t})=m(\boldsymbol{t}) \text { and } r(g \boldsymbol{s}, g \boldsymbol{t})=r(\boldsymbol{s}, \boldsymbol{t}) .
$$

The representation of the covariance of a harmonizable isotropic random field was first obtained by R.J. Swift [4] and more recently by M.M. Rao [3], using a dilation procedure.

Theorem 2.2: The covariance $r: \mathbb{R}^{n} \times \mathbb{R}^{n} \rightarrow \mathbb{C}$ of a weakly harmonizable isotropic random field has representation

$$
r(\boldsymbol{s}, \boldsymbol{t})=2^{\nu} \Gamma\left(\frac{n}{2}\right) \int_{0}^{\infty} \int_{0}^{\infty} \frac{J_{\nu}\left(\left\|\lambda \boldsymbol{s}-\lambda^{\prime} \boldsymbol{t}\right\|\right)}{\left\|\lambda \boldsymbol{s}-\lambda^{\prime} \boldsymbol{t}\right\|^{\nu}} d F\left(\lambda, \lambda^{\prime}\right)
$$

where $J_{\nu}(\cdot)$ is the Bessel function (of the first kind) of order $\nu=(n-2) / 2$ and $F(\cdot, \cdot)$ is of complex bounded Fréchet variation.

Note that when the spectral measure $F(\cdot, \cdot)$ concentrates on the diagonal $\lambda=\lambda^{\prime}$ the representation of the covariance becomes

$$
r(\boldsymbol{s}, \boldsymbol{t})=\tilde{r}(\boldsymbol{s}-\boldsymbol{t})=2^{\nu} \Gamma\left(\frac{n}{2}\right) \int_{0}^{\infty} \frac{J_{\nu}(\lambda \tau)}{(\lambda \tau)^{\nu}} d \tilde{\boldsymbol{F}}(\lambda),
$$


which is the representation of a stationary isotropic covariance obtained by Bochner.

A useful characterization in spherical-polar form for the covariances of harmonizable isotropic random fields was given by Swift [4] as:

Theorem 2.3: A random field $X: \mathbb{R}^{n} \rightarrow L_{0}^{2}(P)$ is weakly harmonizable isotropic iff the covariance function $r(\cdot, \cdot)$ is expressible for $n \geq 2$ as:

$$
r(\boldsymbol{s}, \boldsymbol{t})=\alpha_{n}^{2} \sum_{m=0}^{\infty} \sum_{l=1}^{h(m, n)} S_{m}^{l}(\boldsymbol{u}) S_{m}^{l}(\boldsymbol{v}) \int_{0}^{\infty} \int_{0}^{\infty} \frac{J_{m+\nu}\left(\lambda \tau_{1}\right) J_{m+\nu}\left(\lambda^{\prime} \tau_{2}\right)}{\left(\lambda \tau_{1}\right)^{\nu}\left(\lambda^{\prime} \tau_{2}\right)^{\nu}} d F\left(\lambda, \lambda^{\prime}\right)
$$

where $\nu=\frac{n-2}{2}$ and

(i) $\quad \boldsymbol{s}=\left(\tau_{1}, \boldsymbol{u}\right), \boldsymbol{t}=\left(\tau_{2}, \boldsymbol{v}\right)$ are the spherical polar coordinates of $\boldsymbol{s}, \boldsymbol{t}$ in $\mathbb{R}^{n}$, here $\tau_{1}=\|\boldsymbol{s}\|, \tau_{2}=\|\boldsymbol{t}\|$ and $\boldsymbol{u}=\frac{\boldsymbol{s}}{\tau_{1}}, \boldsymbol{v}=\frac{\boldsymbol{t}}{\tau_{2}}$ are unit vectors. $S_{m}^{l}(\cdot), \quad 1 \leq l \leq h(m, n)=\frac{(2 m+2 \nu)(m+2 \nu-1) !}{(2 \nu) ! m !}, m \geq 1, \quad S_{0}^{l}(\boldsymbol{u})=1$ are the spherical harmonics on the unit $n$-sphere of order $m$.

(iii) $\quad \alpha_{n}>0, \alpha_{n}^{2}=2^{2 \nu+1} \Gamma\left(\frac{n}{2}\right) \pi^{\frac{n}{2}}$ with $F(\cdot, \cdot)$ as a complex function of bounded Fréchet variation.

Using (2) and a form of Karhunen's Theorem, a spectral representation of a harmonizable isotropic random field is given by:

Theorem 2.4: A random field $X: \mathbb{R}^{n} \rightarrow L_{0}^{2}(P)$ is weakly harmonizable isotropic iff it admits a spectral representation of the form

where $Z_{m}^{l}(\cdot)$ satisfies

$$
X(\boldsymbol{t})=\alpha_{n} \sum_{m=0}^{\infty} \sum_{l=1}^{h(m, n)} S_{m}^{l}(\boldsymbol{u}) \int_{0}^{\infty} \frac{J_{m+\nu}(\lambda \tau)}{(\lambda \tau)^{\nu}} d Z_{m}^{l}(\lambda)
$$

$$
E\left(Z_{m}^{l}\left(B_{1}\right) \overline{Z_{m^{\prime}}^{l^{\prime}} B\left({ }_{2}\right)}\right)=\delta_{m m^{\prime}} \delta_{l l^{\prime}} F\left(B_{1}, B_{2}\right)
$$

with $F(\cdot, \cdot)$ a function of bounded Fréchet variation and $\tau=\|\boldsymbol{t}\|, \boldsymbol{u}=\frac{\boldsymbol{t}}{\tau}$, and $\delta_{m m^{\prime}}$ the Kronecker delta, the stochastic integral being in the Dunford-Schwartz sense.

With these details now in place, we will consider a series approximation of a harmonizable isotropic random field.

\section{A Series Approximation}

Recently, Yadrenko and Rakhimov [6], considered a method of simulating a stationary isotropic random field using a stochastic series. With the above background in place, we will consider a similar notion for a harmonizable isotropic random field.

Let $\lambda$ be a nonnegative random variable with density function $f_{1}(\lambda)$ and let $\lambda^{\prime}$ be another nonnegative random variable with density function $f_{2}\left(\lambda^{\prime}\right)$.

Let $\left\{\eta_{m_{1}}\right\}$ and $\left\{\eta_{m_{2}}\right\}$ be two mutually independent sequences of independent random variables such that for each $m$ 
and

$$
E\left(\eta_{m_{1}}\right)=E\left(\eta_{m_{2}}\right)=0
$$

$$
E\left(\eta_{m_{i}} \eta_{s_{j}}\right)=\delta_{m}^{s} \delta_{i}^{j}
$$

where $\delta_{i}^{j}$ is the Kronecker delta.

Let $\boldsymbol{t} \in \mathbb{R}^{2}$, with polar coordinates $(\|\boldsymbol{t}\|, \theta)$ and consider the stochastic series

$$
X(\boldsymbol{t})=X(\|\boldsymbol{t}\|, \theta)=\sum_{m=0}^{\infty} \sqrt{\nu_{m}} J_{m}(\lambda\|\boldsymbol{t}\|)\left[\eta_{m_{1}} \cos m \theta+\eta_{m_{2}} \sin m \theta\right]
$$

where

$$
\nu_{m}= \begin{cases}1 & \text { for } m=0 \\ 2 & \text { for } m \neq 0\end{cases}
$$

Now using (3), the covariance of the series is computed as

$$
\begin{aligned}
r(\boldsymbol{s}, \boldsymbol{t})= & E(X(\boldsymbol{s}) X(\boldsymbol{t})) \\
= & E\left(X\|\boldsymbol{s}\|, \theta_{1}\right) X\left(\|\boldsymbol{t}\|, \theta_{2}\right) \\
= & E\left(\sum_{m=0}^{\infty} \sqrt{\nu_{m}} J_{m}(\lambda\|\boldsymbol{s}\|)\left[\eta_{m_{1}} \cos m \theta_{1}+\eta_{m_{2}} \sin m \theta_{1}\right]\right. \\
& \left.\quad \times \sum_{m=0}^{\infty} \sqrt{\nu_{m}} J_{m}\left(\lambda^{\prime}\|\boldsymbol{t}\|\right)\left[\eta_{m_{1}} \cos m \theta_{2}+\eta_{m_{2}} \sin m \theta_{2}\right]\right) .
\end{aligned}
$$

Thus

$$
\begin{gathered}
r(\boldsymbol{s}, \boldsymbol{t})=E\left(J _ { 0 } \left(( \lambda \| \boldsymbol { s } \| ) \eta _ { 0 1 } J _ { 0 } \left(\left(\lambda^{\prime}\|\boldsymbol{t}\|\right) \eta_{01}+2 \sum_{m=0}^{\infty} J_{m}(\lambda\|\|) J_{m}\left(\lambda^{\prime}\|\boldsymbol{t}\|\right)\right.\right.\right. \\
\times\left[\eta_{m_{1}} \cos m \theta_{1}+\eta_{m_{2}} \sin m \theta_{1}\right]\left[\eta_{m_{1}} \cos m \theta_{2}+\eta_{m_{2}} \sin m \theta_{2}\right] \\
\left.+2 \sum \sum_{k \neq l} J_{k}(\lambda\|\boldsymbol{s}\|) J_{l}\left(\lambda^{\prime}\|\boldsymbol{t}\|\right)\left[\eta_{k_{1}} \cos k \theta_{1}+\eta_{k_{2}} \sin k \theta_{1}\right]\left[\eta_{l_{1}} \cos l \theta_{2}+\eta_{l_{2}} \sin l \theta_{2}\right]\right),
\end{gathered}
$$

upon applying the expectation, the expression becomes

$$
\begin{gathered}
r(\boldsymbol{s}, \boldsymbol{t})=\int_{0}^{\infty} \int_{0}^{\infty}\left[J _ { 0 } \left(( \lambda \| \boldsymbol { s } \| ) J _ { 0 } \left(\left(\lambda^{\prime}\|\boldsymbol{t}\|\right)\right.\right.\right. \\
\left.\left.+2 \sum_{m=0}^{\infty} J_{m}(\lambda\|\boldsymbol{s}\|) J_{m}\left(\lambda^{\prime}\|\boldsymbol{t}\|\right) \cos m\left(\theta_{1}-\theta_{2}\right)\right) f_{1}(\lambda) f_{2}\left(\lambda^{\prime}\right)\right] d \lambda d \lambda^{\prime}
\end{gathered}
$$

where $f_{1}(\cdot)$ and $f_{2}(\cdot)$ are densities of the nonnegative random variables $\lambda$ and $\lambda^{\prime}$, respectively.

The following lemma, the proof of which was given by Swift [4], 
Lemma 3.1: With the notation given in Theorem 2.3, one has

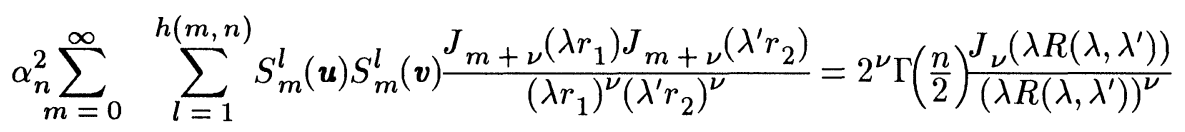

where $R\left(\lambda, \lambda^{\prime}\right)=\left(r_{1}^{2}+\left(\frac{\lambda^{\prime}}{\lambda}\right)^{2} r_{2}^{2}-2\left(\frac{\lambda^{\prime}}{\lambda}\right) r_{1} r_{2} \cos \theta\right)^{\frac{1}{2}}$ and $\cos \theta=\boldsymbol{u} \cdot \boldsymbol{v}$, the angle between the unit vectors $\boldsymbol{u}, \boldsymbol{v}$, reduces for $n=2$, to

$$
2 \pi \sum_{m=0}^{\infty} \sum_{l=1}^{2} S_{m}^{l}(\boldsymbol{u}) S_{m}^{l}(\boldsymbol{v}) J_{m}\left(\lambda r_{1}\right) J_{m}\left(\lambda^{\prime} r_{2}\right)=J_{0}\left(\sqrt{\lambda^{2} r_{1}^{2}+\left(\lambda^{\prime}\right)^{2} r_{2}^{2}-2 \lambda \lambda^{\prime} \cos \theta}\right) .
$$

Substituting expression (6) into (4) gives the covariance as

$$
\begin{gathered}
r(\boldsymbol{s}, \boldsymbol{t})=E\left(X\left(\|\boldsymbol{s}\|, \theta_{1}\right) X\left(\|\boldsymbol{t}\|, \theta_{2}\right)\right) \\
=2 \pi \int_{0}^{\infty} \int_{0}^{\infty} J_{0}\left(\sqrt{\lambda^{2}\|\boldsymbol{s}\|^{2}+\left(\lambda^{\prime}\right)^{2}\|\boldsymbol{t}\|^{2}-2 \lambda \lambda^{\prime} \cos \left(\theta_{1}-\theta_{2}\right)}\right) f_{1}(\lambda) f_{2}\left(\lambda^{\prime}\right) d \lambda d \lambda^{\prime} .
\end{gathered}
$$

But (7) is just the representation of the covariance of a strongly harmonizable isotropic random field on the plane $\mathbb{R}^{2}$, with spectral density

$$
f\left(\lambda, \lambda^{\prime}\right)=f_{1}(\lambda) f_{2}\left(\lambda^{\prime}\right)
$$

This observation is summarized as:

Theorem 3.1: The two-dimensional random field $X: \mathbb{R}^{2} \rightarrow L^{2}(P)$ with representation

$$
X(\boldsymbol{t})=X(\|\boldsymbol{t}\|, \theta)=\sum_{m=0}^{\infty} \sqrt{\nu_{m}} J_{m}(\lambda\|\boldsymbol{t}\|)\left[\eta_{m_{1}} \operatorname{cosm} \theta+\eta_{m_{2}} \operatorname{sinm} \theta\right]
$$

where

$$
\nu_{m}= \begin{cases}1 & \text { for } m=0 \\ 2 & \text { for } m \neq 0\end{cases}
$$

is strongly harmonizable isotropic with zero mean and a covariance with representation given by $(7)$.

To approximate a strongly harmonizable isotropic random field, the convergence of the partial sum

$$
X_{N}(\boldsymbol{t})=X_{N}(\|\boldsymbol{t}\|, \theta)=\sum_{m=0}^{N} \sqrt{\nu_{m}} J_{m}(\lambda\|\boldsymbol{t}\|)\left[\eta_{m_{1}} \cos m \theta+\eta_{m_{2}} \sin m \theta\right]
$$

will be considered. Thus

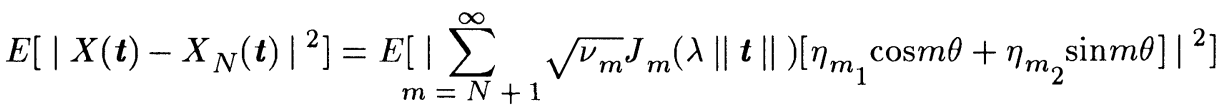

$$
\begin{aligned}
& =2 \int_{0}^{\infty} \int_{0}^{\infty} \sum_{m=N+1}^{\infty}\left|J_{m}(\lambda\|\boldsymbol{t}\|) J_{m}\left(\lambda^{\prime}\|\boldsymbol{t}\|\right)\right| f_{1}(\lambda) f_{2}\left(\lambda^{\prime}\right) d \lambda d \lambda^{\prime} .
\end{aligned}
$$


But, the growth inequality (cf., Lebedev [2])

$$
\left|J_{m}(z)\right| \leq \frac{|z|}{m}
$$

gives that

$$
\begin{gathered}
E\left[\left|X(\boldsymbol{t})-X_{N}(\boldsymbol{t})\right|^{2}\right] \leq 2 \int_{0}^{\infty} \int_{0}^{\infty} \sum_{m=N+1}^{\infty} \lambda \lambda^{\prime} \frac{\|\boldsymbol{t}\|^{2}}{m^{2}} f_{1}(\lambda) f_{2}\left(\lambda^{\prime}\right) d \lambda d \lambda^{\prime} \\
\leq \frac{2\|\boldsymbol{t}\|^{2}}{N} \int_{0}^{\infty} \int_{0}^{\infty} \lambda \lambda^{\prime} f_{1}(\lambda) f_{2}\left(\lambda^{\prime}\right) d \lambda d \lambda^{\prime}
\end{gathered}
$$

Thus, the error in approximating the random field (3) by its partial sum depends upon a boundedness condition upon its moments and is given by:

Theorem 3.2: Suppose that the two-dimensional random field $X(\cdot)$ with representation

$$
X(\boldsymbol{t})=X(\|\boldsymbol{t}\|, \theta)=\sum_{m=0}^{\infty} \sqrt{\nu_{m}} J_{m}(\lambda\|\boldsymbol{t}\|)\left[\eta_{m_{1}} \cos m+\eta_{m_{2}} \operatorname{sinm} \theta\right]
$$

satisfies

$$
\int_{0}^{\infty} \int_{0}^{\infty} \lambda \lambda^{\prime} f_{1}(\lambda) f_{2}\left(\lambda^{\prime}\right) d \lambda d \lambda^{\prime} \leq \infty
$$

then the partial sum $X_{N}(\boldsymbol{t})$, given by (8), converges in the $L^{2}$-sense to $X(\boldsymbol{t})$ as $N \rightarrow \infty$.

Letting

$$
\mu_{j, k}=\int_{0}^{\infty} \int_{0}^{\infty} \lambda^{j}\left(\lambda^{\prime}\right)^{k} d F\left(\lambda, \lambda^{\prime}\right)
$$

then

Corollary 3.1: The error of the $L^{2}$-approximation of the random field (3) by its partial sum (8) is bounded by $\left(2\|\boldsymbol{t}\|^{2} \mu_{1,1}\right) / N$.

In practice, it is often desirable to guarantee not only the closeness of the partial sum, but also the closeness of partial derivatives up to a given order. The following theorem is stated here for completeness. The proof follows using similar, (though tedious), computations as above and the inequality (cf., Lebedev [2])

$$
\left(J_{m}^{\prime}(z)\right)^{2} \leq \frac{1}{2}\left(J_{m-1}^{2}(z)+J_{m+1}^{2}(z)\right) .
$$

Theorem 3.3: $\quad$ Consider the two-dimensional random field $X(\cdot)$ with representation

$$
X(\boldsymbol{t})=X(\|\boldsymbol{t}\|, \theta)=\sum_{m=0}^{\infty} \sqrt{\nu_{m}} J_{m}(\lambda\|\boldsymbol{t}\|)\left[\eta_{m_{1}} \operatorname{cosm} \theta+\eta_{m_{2}} \operatorname{sinm} \theta\right] .
$$

1. If $\mu_{2,2} \leq \infty$ and $N>1$, then

$$
E\left[\left|\frac{\partial X(\|\boldsymbol{t}\|, \theta)}{\partial\|\boldsymbol{t}\|}-\frac{\partial X_{N}(\|\boldsymbol{t}\|, \theta)}{\partial\|\boldsymbol{t}\|}\right|^{2}\right] \leq \frac{2\|\boldsymbol{t}\|^{2} \mu_{2,2}}{N-1}
$$


and

$$
E\left[\left|\frac{\partial X(\|\boldsymbol{t}\|, \theta)}{\partial \theta}-\frac{\partial X_{N}(\|\boldsymbol{t}\|, \theta)}{\partial \theta}\right|^{2}\right] \leq \frac{2\|\boldsymbol{t}\|^{4} \mu_{2,2}}{N-1}
$$

2. If $\mu_{2,2} \leq \infty$, and $\mu_{3,3} \leq \infty$ and $N>2$, then

$$
\begin{gathered}
E\left[\left|\frac{\partial^{2} X(\|\boldsymbol{t}\|, \theta)}{\partial^{2}\|\boldsymbol{t}\|}-\frac{\partial^{2} X_{N}(\|\boldsymbol{t}\|, \theta)}{\partial^{2}\|\boldsymbol{t}\|}\right|^{2}\right] \leq \frac{2\|\boldsymbol{t}\|^{2} \mu_{3,3}}{N-2} \\
E\left[\left|\frac{\partial^{2} X(\|\boldsymbol{t}\|, \theta)}{\partial^{2} \theta}-\frac{\partial^{2} X_{N}(\|\boldsymbol{t}\|, \theta)}{\partial^{2} \theta}\right|^{2}\right] \leq \frac{2\|\boldsymbol{t}\|^{4}\left(\|\boldsymbol{t}\|^{2} \mu_{3,3}+\mu_{2,2}\right)}{N-2}
\end{gathered}
$$

and

$$
E\left[\left|\frac{\partial^{2} X(\|\boldsymbol{t}\|, \theta)}{\partial\|\boldsymbol{t}\| \partial \theta}-\frac{\partial^{2} X_{N}(\|\boldsymbol{t}\|, \theta)}{\partial\|\boldsymbol{t}\| \partial \theta}\right|^{2}\right] \leq \frac{2\|\boldsymbol{t}\|^{2}\left(\|\boldsymbol{t}\|^{4} \mu_{2,2}+\mu_{1,1}\right)}{N-2}
$$

\section{Acknowledgments}

The author expresses his thanks to Professor M.M. Rao for his continuing advice, encouragement and guidance during the work of this project. The author also expresses his gratitude to Western Kentucky University for a sabbatical leave during the Fall 1998 semester and for release time during Spring 1999 semester, during which this work was completed. The generous support of the Committee for Faculty Research at Western Kentucky University is also acknowledged. Without their support, my travels to India would not have been possible.

\section{References}

[1] Chang, D.K. and Rao, M.M., Bimeasures and nonstationary processes, Real and Stochastic Analysis, John Wiley and Sons, New York (1986), 7-118.

[2] Lebedev, N.N., Special Functions and Their Applications, Dover Publications, New York 1972.

[3] Rao, M.M., Characterization of isotropic harmonizable covariances and related representations, (to appear).

[4] Swift, R., The structure of harmonizable isotropic random fields, Stoch. Anal. and Appl. 12 (1994), 583-616.

[5] Swift, R., Some aspects of harmonizable processes and fields, In: Real and Stoch. Analysis: Recent Advances (ed. by M.M. Rao), CRC Press, Boca Raton, FL (1997), 303-365.

[6] Yadrenko, M.I. and Rakhimov, A.K., Statistical Simulation of a Homogeneous Isotropic Random Field on the Plane and Estimations of Simulation Errors, Theory Prob. and Math. Statist. 491994. 


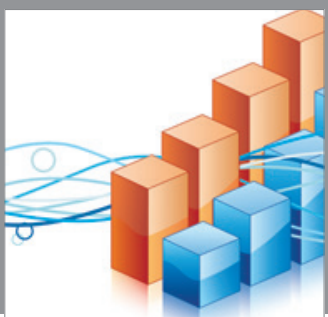

Advances in

Operations Research

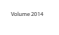

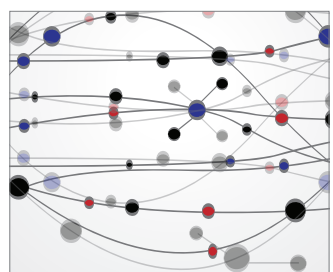

\section{The Scientific} World Journal
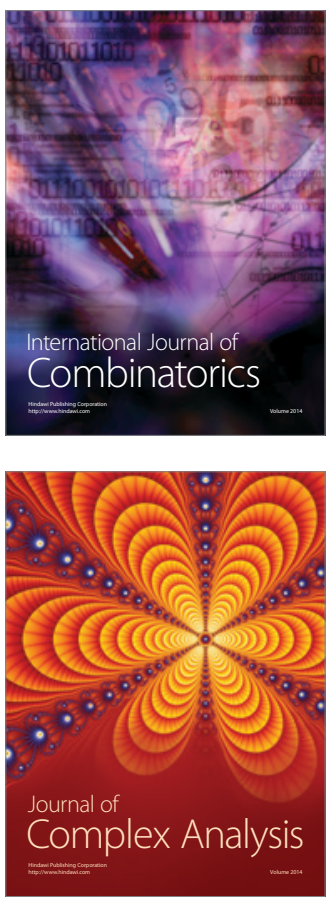

International Journal of

Mathematics and

Mathematical

Sciences
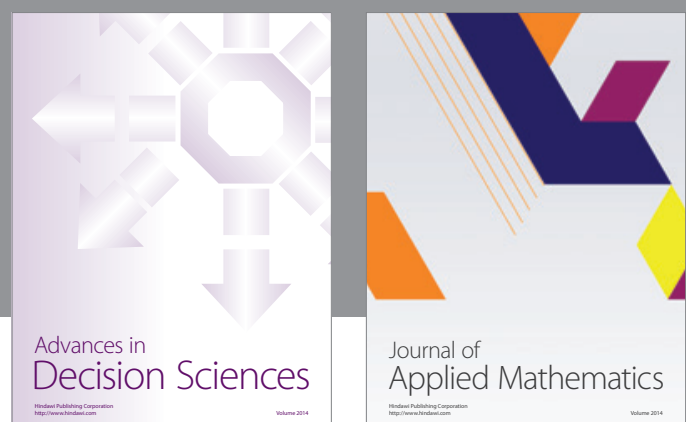

Journal of

Applied Mathematics
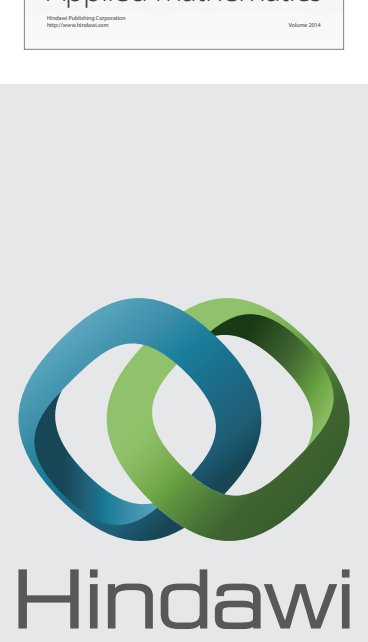

Submit your manuscripts at http://www.hindawi.com
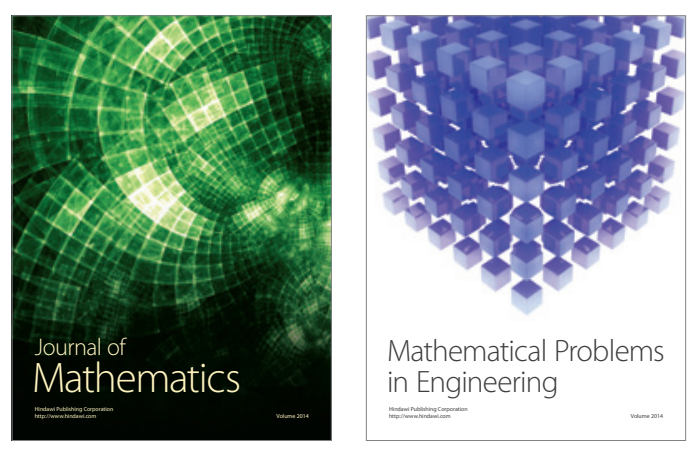

Mathematical Problems in Engineering
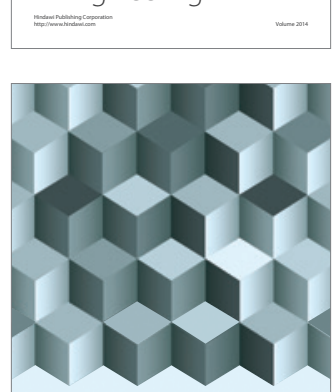

Journal of

Function Spaces
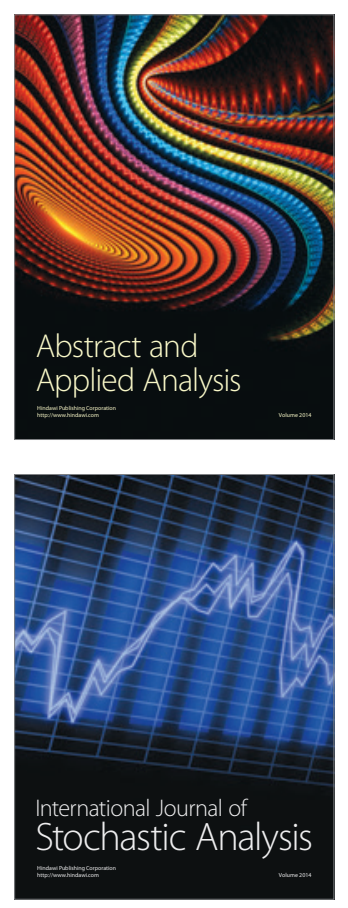

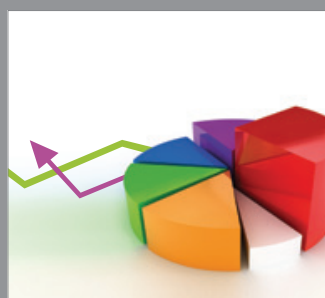

ournal of

Probability and Statistics

Promensencen
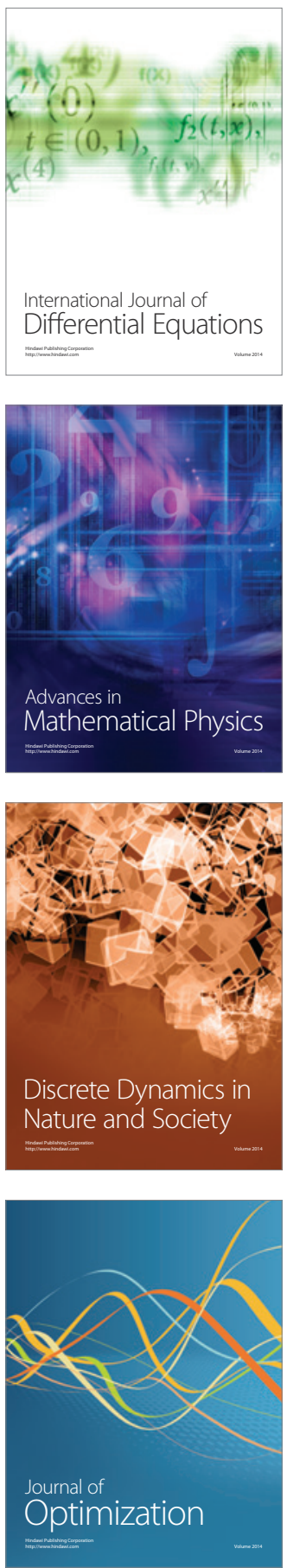